\title{
SEMI-DISCRÉTISATION EN ESPACE DU PROBLÈME DE LA STABILISATION INTERNE DE L'ÉQUATION DES POUTRES
}

\author{
KARIM RAmdani, TAKÉO TAKahashi And Marius TuCsnaK ${ }^{1}$
}

\begin{abstract}
We consider the finite-difference space semi-discretization of the beam equation with an internal stabilization. By using a frequency domain approach, we prove that this scheme yields a uniform exponential decay rate (with respect to the mesh size). This positive result is in contrast with previous negative ones for the case of boundary stabilization or for other evolution equations.

Résumé. On s'intéresse à la semi-discrétisation en espace de l'équation des poutres avec dissipation interne par différences finies. En utilisant une approche fréquentielle, on prouve que le schéma construit conduit à un taux de décroissance exponentielle de l'énergie qui est uniforme (vis à vis du pas de discrétisation). Ce résultat positif est à comparer aux résultats négatifs précédemment établis pour le cas de la stabilisation frontière ou pour d'autres équations d'évolution.
\end{abstract}

\section{INTRODUCTION}

Cet article est consacré à l'étude de l'approximation numérique du problème de la stabilisation interne de l'équation des poutres. On suppose que la poutre est soumise à une force distribuée de type feedback (stabilisation interne). On considère l'approximation usuelle du problème par un schéma aux différences finies semi-discrétisé en espace. On montre qu'un tel schéma conduit à des solutions approchées vérifiant une propriété de décroissance exponentielle uniforme de l'énergie (vis à vis du pas de discrétisation $h$ ).

A première vue, ce résultat peut paraître très naturel. Il l'est moins si on le compare aux nombreux résultats négatifs établis pour des problèmes similaires. En effet, il s'avère que des discrétisations usuelles par différences ou éléments finis de problèmes de stabilisation interne ou frontière conduisent à des schémas ne fournissant pas des taux de décroissance exponentielle uniformes de l'énergie. Ce phénomène est directement lié à l'apparition lors de la discrétisation numérique de modes parasites hautes fréquences que le terme de dissipation n'est pas à même de stabiliser. L'apparition de tels modes parasites a été soulignée dans de nombreux travaux (voir, par exemple, Glowinski, Li et Lions [2], Infante et Zuazua [3], Tébou et Zuazua [13]) et de nombreuses méthodes ont été proposées pour remédier à ce problème. Parmi les principales solutions retenues, on peut citer la régularisation de Tychonoff [2], les éléments finis mixtes dans Banks, Ito, et Wang [1], le filtrage des hautes fréquences dans [3] et dans Micu [8], et l'utilisation d'une viscosité numérique artificielle dans Ramdani, Takahashi et Tucsnak [11] et dans [13].

Soulignons enfin que la question de la semi-discrétisation du problème de la contrôlabilité frontière de l'équation des poutres a été abordée dans León et Zuazua [4]. En particulier, il y est établi que l'on perd le caractère uniforme de l'observabilité pour des schémas aux différences finies usuels. Enfin, dans Ramdani, Takahashi et Tucsnak [12], nous étudions un problème similaire à celui traité ici, à savoir celui de la stabilisation interne de l'équation des plaques dans un domaine carré. Nous démontrons en particulier que moyennant

\footnotetext{
1 INRIA (Projet Corida) et IECN, Université Nancy 1, BP 239, Vandœuvre-lès-Nancy, 54506, France
}

(C) EDP Sciences, SMAI 2007 
l'introduction d'un terme de viscosité numérique approprié, on obtient un schéma uniformément exponentiellement stable. Toutefois, soulignons que la question de savoir si ce terme est nécessaire pour assurer la stabilité exponentielle uniforme reste une question ouverte. Ceci provient notamment du fait que les deux ingrédients essentiels que sont la condition de gap uniforme et la condition d'observabilité uniforme établis dans la Proposition 2.1 ne sont plus valables en dimension supérieure à un.

\section{1. ÉNONCÉ DU RÉSULTAT PRINCIPAL}

Considérons le problème aux limites et aux conditions initiales suivant:

$$
\begin{gathered}
\frac{\partial^{2} w}{\partial t^{2}}+\frac{\partial^{4} w}{\partial x^{4}}+\chi_{[a, b]} \frac{\partial w}{\partial t}=0, \quad 0<x<\pi, t>0, \\
w(0, t)=w(\pi, t)=0 \quad \forall t>0 \\
\frac{\partial^{2} w}{\partial x^{2}}(0, t)=\frac{\partial^{2} w}{\partial x^{2}}(\pi, t)=0 \quad \forall t>0 \\
w(x, 0)=w_{0}(x), \quad \frac{\partial w}{\partial t}(x, 0)=w_{1}(x) \quad \forall x \in(0, \pi),
\end{gathered}
$$

où $\chi_{[a, b]}$ désigne la fonction caractéristique de l'intervalle $\left.[a, b] \subset\right] 0, \pi[$.

On rappelle que l'on a le résultat de stabilité exponentielle suivant :

Théorème 1.1. On suppose que $w_{0} \in H^{2}(0, \pi) \cap H_{0}^{1}(0, \pi)$ et que $w_{1} \in L^{2}(0, \pi)$. Alors, le système (1)-(4) admet une unique solution $w \in C\left(0, \infty ; H^{2}(0, \pi) \cap H_{0}^{1}(0, \pi)\right) \cap C^{1}\left(0, \infty ; H_{0}^{1}(0, \pi)\right)$. De plus, le système (1)-(4) est exponentiellement stable, i.e. il existe des constantes $M_{0}, \alpha_{0}>0$ ne dépendant que de a, b telles que

$$
\left(\|\dot{w}(t)\|_{L^{2}(0, \pi)}^{2}+\|w(t)\|_{H^{2}(0, \pi)}^{2}\right) \leq M_{0} e^{-\alpha_{0} t}\left(\left\|w_{1}\right\|_{L^{2}(0, \pi)}^{2}+\left\|w_{0}\right\|_{H^{2}(0, \pi)}^{2}\right) \quad \forall t>0 .
$$

Une question se pose naturellement lorsque l'on veut discrétiser le système (1)-(4) : peut-on construire des schémas générant des solutions dont l'énergie discrète décroît exponentiellement et uniformément vis à vis du pas de discrétisation? Nous allons voir que la réponse à cette question est positive dans le cas d'une semidiscrétisation usuelle par différences finies. Pour ce faire, commençons par la description précise du schéma retenu. Étant donné $N \in \mathbb{N}$, on pose

$$
h=\frac{\pi}{N+1}
$$

et on suppose, sans perte de généralité, qu'il existe des entiers $a(h), b(h) \in\{1, \ldots, N\}$ tels que

$$
a=a(h) h, \quad b=b(h) h,
$$

Posons

$$
V_{h}=\mathbb{R}^{N}
$$

Pour tout $j \in\{0, \ldots, N+1\}$, on désigne par $w_{j}$ une approximation de la solution $w$ du système (1)-(4) au point $x_{j}=j h$ et par $w_{h} \in V_{h}$ le vecteur de composantes $w_{j}$ pour $1 \leq j \leq N$. On utilise l'approximation aux différences finies centrée usuelle du Laplacien :

$$
\Delta w(j h) \approx \frac{1}{h^{2}}\left(w_{j+1}+w_{j-1}-2 w_{j}\right)
$$

Afin de satisfaire les conditions aux limites (3), on pose

$$
w_{0}=0, w_{N+1}=0, w_{-1}=-w_{1}, w_{N+2}=-w_{N} .
$$


On définit alors la matrice $A_{0 h}$ du Bilaplacien discrétisé par l'intermédiaire de sa racine carrée $A_{0 h}^{\frac{1}{2}}$ :

$$
\left(A_{0 h}^{\frac{1}{2}} w_{h}\right)_{j}=-\frac{1}{h^{2}}\left(w_{j+1}+w_{j-1}-2 w_{j}\right), \quad \forall 1 \leq j \leq N .
$$

Le schéma aux différences finies pour le système (1)-(4) étudié dans cet article s'écrit alors

$$
\begin{gathered}
\ddot{w}_{j}+\left(A_{0 h} w_{h}\right)_{j}+\left(\chi_{\mathcal{O}} \dot{w}_{h}\right)_{j}=0, \quad 1 \leq j \leq N, t>0, \\
w_{h}(0)=w_{0 h}, \quad \dot{w}_{h}(0)=w_{1 h} .
\end{gathered}
$$

Dans (7), $w_{0 h}$ et $w_{1 h}$ désignent respectivement des approximations des valeurs de $w_{0}$ et $w_{1}$ aux points de la discrétisation. Dans (6), le teme $\chi_{\mathcal{O}} \dot{w}_{h}$ désigne le vecteur de $V_{h}$ de composantes

$$
\left(\chi_{\mathcal{O}} \dot{w}_{h}\right)_{j}= \begin{cases}\dot{w}_{j} & \text { si } a(h) \leq j \leq b(h), \\ 0 & \text { sinon. }\end{cases}
$$

Le résultat principal de cet article est le suivant.

Théorème 1.2. La famille de systèmes définis par les relations (6)-(7) est uniformément exponentiellement stable, au sens où il existe des constantes $M, \alpha, h^{*}>0$ (indépendantes de $h, w_{0 h}$ et de $w_{1 h}$ ) telles que pour tout $h \in\left(0, h^{*}\right)$ :

$$
\left(\left\|\dot{w}_{h}(t)\right\|^{2}+\left\|A_{0 h}^{\frac{1}{2}} w_{h}(t)\right\|^{2}\right) \leq M e^{-\alpha t}\left(\left\|w_{1 h}\right\|^{2}+\left\|A_{0 h}^{\frac{1}{2}} w_{0 h}\right\|^{2}\right) \quad \forall t>0 .
$$

Ci-dessus ainsi que dans le reste de l'article, on notera par $\|\cdot\|$ la norme Euclidienne dans $\mathbb{R}^{m}$ pour différentes valeurs de $m$.

\section{DÉMONSTRATION DU RÉSUlTAT PRINCIPAL}

\subsection{Résultats préliminaires}

Avant d'établir le Théorème 1.2, on introduit des notations supplémentaires. Rappelons que $V_{h}=\mathbb{R}^{N}$ et introduisons

$$
U_{h}=\mathbb{R}^{(b(h)-a(h)+1)}
$$

l'espace de la commande discrétisée, où les entiers $a(h)$ and $b(h)$ sont définis par (5). On introduit l'opérateur de contrôle $B_{0 h} \in \mathcal{L}\left(U_{h}, V_{h}\right)$ défini pour tout $u_{h} \in U_{h}$ par

$$
\left(B_{0 h} u_{h}\right)_{j}= \begin{cases}u_{j} & \text { si } a(h) \leq j \leq b(h), \\ 0 & \text { sinon. }\end{cases}
$$

L'adjoint $B_{0 h}^{*} \in \mathcal{L}\left(V_{h}, U_{h}\right)$ de $B_{0 h}$ est alors défini pour tout $w_{h} \in V_{h}$ par

$$
\left(B_{0 h}^{*} w_{h}\right)_{j}=w_{j}, \quad a(h) \leq j \leq b(h) .
$$

La semi-discrétisation (6)-(7) s'écrit alors

$$
\begin{gathered}
\ddot{w}_{j}+\left(A_{0 h} w_{h}\right)_{j}+\left(B_{0 h} B_{0 h}^{*} \dot{w}_{h}\right)_{j}=0, \quad 1 \leq j \leq N, \\
w_{j}=\left(A_{0 h}^{\frac{1}{2}} w_{h}\right)_{j}=0, \quad j=0, N+1
\end{gathered}
$$




$$
w_{j}(0)=w_{0 h}, \quad \dot{w}_{j}(0)=w_{1 h}, \quad 0 \leq j \leq N+1 .
$$

On vérifie sans peine que la suite $\left(\left\|B_{0 h}\right\|_{\mathcal{L}\left(U_{h}, V_{h}\right)}\right)$ est bornée et que les valeurs propres de $A_{0 h}^{\frac{1}{2}}$ sont (voir par exemple [14])

$$
\lambda_{p, h}=\frac{4}{h^{2}} \sin ^{2}\left(\frac{p h}{2}\right), \quad 1 \leq p \leq N .
$$

Une suite de vecteurs propres normalisés dans $V_{h}$ associés à ces valeurs propres est

$$
\varphi_{p, h}=\left(\varphi_{p, h}^{j}\right)_{1 \leq j \leq N}, \quad \varphi_{p, h}^{j}=\sqrt{\frac{2 h}{\pi}} \sin (j p h)
$$

La démonstration du Théorème 1.2 repose sur la caracterisation fréquentielle suivante de la stabilité exponentielle uniforme d'une famille de semigroupes (voir [7, p.162]).

Théorème 2.1. Soit $\left(\mathbb{T}_{h}\right)_{h>0}$ une famille de semigroupes de contractions sur les espaces de Hilbert $V_{h}$ et soit $A_{h}$ le générateur infinitésimal correspondant. La famille $\left(\mathbb{T}_{h}\right)_{h>0}$ est uniformément exponentiellement stable si et seulement si les deux conditions suivantes sont satisfaites:

(i) Pour tout $h>0, i \mathbb{R} \subset \rho\left(A_{h}\right)$, où $\rho\left(A_{h}\right)$ désigne l'ensemble résolvant de $A_{h}$,

(ii) $\sup _{h>0, \omega \in \mathbb{R}}\left\|\left(i \omega-A_{h}\right)^{-1}\right\|<+\infty$.

\subsection{Démonstration $\mathbf{d u}$ Théorème $\mathbf{1 . 2}$}

Afin d'appliquer le Théorème 2.1, on réécrit le système (10)-(12) sous la forme d'un système d'ordre un. On introduit à cet effet l'espace produit $X_{h}=V_{h} \times V_{h}$, et on le munit de la norme

$$
\left\|\left(\varphi_{h}, \psi_{h}\right)\right\|_{X_{h}}^{2}=\left\|A_{0 h}^{\frac{1}{2}} \varphi_{h}\right\|^{2}+\left\|\psi_{h}\right\|^{2}
$$

Posant $z_{h}=\left[\begin{array}{c}w_{h} \\ \dot{w}_{h}\end{array}\right]$, les équations (10)-(12) s'écrivent alors sous la forme équivalente

$$
\dot{z}_{h}(t)=A_{h} z_{h}(t), \quad z_{h}(0)=z_{0}
$$

où $z_{0 h}=\left[\begin{array}{l}w_{0 h} \\ w_{1 h}\end{array}\right]$ et $A_{h} \in \mathcal{L}\left(X_{h}\right)$ est défini par

$$
A_{h}=\left[\begin{array}{cc}
0 & I \\
-A_{0 h} & -B_{0 h} B_{0 h}^{*}
\end{array}\right]
$$

Il est utile pour la suite de la preuve d'introduire l'opérateur $A_{1 h}$

$$
A_{1 h}=\left[\begin{array}{cc}
0 & I \\
-A_{0 h} & 0
\end{array}\right] \in \mathcal{L}\left(X_{h}\right)
$$

de sorte que

$$
A_{h}=A_{1 h}-\left[\begin{array}{cc}
0 & 0 \\
0 & B_{0 h} B_{0 h}^{*}
\end{array}\right] .
$$

Dans la suite, on aura recours aux éléments propres de l'opérateur $A_{1 h}$. Si l'on étend la définition de $\lambda_{m, h}$ et de $\varphi_{m, h}$ aux valeurs de $m \in\{-1, \ldots,-N\}$ en posant

$$
\left\{\begin{array}{l}
\lambda_{m, h}=-\lambda_{-m, h} \\
\varphi_{m, h}=\varphi_{-m, h}
\end{array}\right.
$$


alors on vérifie aisément qu'une base orthonormée de $X_{h}$ formée de vecteurs propres de $A_{1 h}$ est donnée par

$$
\Phi_{m, h}=\frac{1}{\sqrt{2}}\left[\begin{array}{c}
-\frac{i}{\lambda_{m, h}} \varphi_{m, h} \\
\varphi_{m, h}
\end{array}\right], \quad 1 \leq|m| \leq N,
$$

$\Phi_{m, h}$ étant un vecteur propre associé à la valeur propre $i \lambda_{m, h}$.

La preuve du Théorème 1.2 utilise de manière déterminante les deux ingrédients que sont la condition de gap uniforme et la condition d'observabilité uniforme suivantes.

Proposition 2.1. Avec les notations ci-dessus, il existe $h^{*}>0, \gamma>0$ et $\beta>0$ tels que, pour tout $0<h<h^{*}$ et tout $n \in\{1,2, \ldots, N\}$ :

$$
\lambda_{n+1, h}-\lambda_{n, h} \geq \gamma,
$$

et

$$
\left\|B_{0 h}^{*} \varphi_{n, h}\right\| \geq \beta .
$$

Démonstration.

(1) Démonstration de (20): grâce à la relation (13) il vient que

$$
\begin{aligned}
\lambda_{p+1, h}-\lambda_{p, h} & =\frac{4}{h^{2}}\left(\sin ^{2}\left(\frac{(p+1) h}{2}\right)-\sin ^{2}\left(\frac{p h}{2}\right)\right) \\
& =\frac{4}{h^{2}} \sin \left((2 p+1) \frac{h}{2}\right) \sin \left(\frac{h}{2}\right) \\
& \geq \frac{4}{h^{2}} \sin ^{2}\left(\frac{h}{2}\right) \\
& \geq \gamma
\end{aligned}
$$

pour $h<h^{*}$ et $h^{*}$ suffisamment petit.

(2) Démonstration de (21): en utilisant les relations (14) et (9), on obtient que

$$
\left\|B_{0 h}^{*} \varphi_{n, h}\right\|^{2}=\sum_{j=a(h)}^{b(h)} \frac{2 h}{\pi} \sin ^{2}(j n h) .
$$

On commence par remarquer que compte tenu de la symétrie de $\left\|B_{0 h}^{*} \varphi_{n, h}\right\|^{2}$ vis à vis du changement de variable $n \mapsto N+1-n$, on peut se contenter d'étudier cette fonction pour $n \leq \frac{N+1}{2}$. On note ensuite que la somme ci-dessus peut être vue comme une somme de Riemann correspondant à l'intégrale $\int_{a}^{b} \sin ^{2}(n x) d x$, pour laquelle on vérifie facilement qu'il existe $\delta>0$ tel que

$$
\int_{a}^{b} \sin ^{2}(n x) d x>\delta \quad \forall n \in \mathbb{N}^{*}
$$

Plus précisément, on a

$$
\left(\int_{a}^{b} \sin ^{2}(n x) d x\right)-\sum_{j=a(h)}^{b(h)} h \sin ^{2}(j n h)=\sum_{k=a(h)}^{b(h)-1} \int_{k h}^{(k+1) h}\left(\sin ^{2}(n x)-\sin ^{2}(n k h)\right) d x+h \sin ^{2}(b n)
$$


Le théorème des accroissements finis montre alors que

$$
\left|\left(\int_{a}^{b} \sin ^{2}(n x) d x\right)-\sum_{j=a(h)}^{b(h)} h \sin ^{2}(j n h)\right| \leq(b-a) n h+h .
$$

Par conséquent, pour $h^{*}$ assez petit, il existe $\varepsilon>0$ (ne dépendant que de $\delta$, a et $b$ ) tel que pour $n h<\varepsilon$ (autrement dit pour les entiers $n$ situés en basse fréquence), on a

$$
\sum_{j=a(h)}^{b(h)} h \sin ^{2}(j n h) \geq \frac{\delta}{2}
$$

Pour les entiers $n$ situés en haute fréquence $(n h \geq \varepsilon)$, on exploite le fait que l'on peut calculer directement la somme dans (22):

$$
\sum_{j=a(h)}^{b(h)} 2 \sin ^{2}(j n h)=(b(h)-a(h)+1)-\left(\cos (n h(b(h)+a(h))) \frac{\sin (n h(b(h)-a(h)+1))}{\sin (n h)}\right)
$$

et donc, en posant

$$
i(h)=\frac{b-a+h}{\pi}>\frac{b-a}{\pi}
$$

on a pour $\varepsilon \leq n h \leq \frac{N+1}{2} h=\frac{\pi}{2}$

$$
\begin{aligned}
\sum_{j=a(h)}^{b(h)} \frac{2 h}{\pi} \sin ^{2}(j n h) & \geq i(h)-\frac{h}{\pi \sin (n h)} \\
& \geq \frac{b-a}{\pi}-\frac{h}{\pi \sin (\varepsilon)} .
\end{aligned}
$$

Par suite, pour $h^{*}$ suffisamment petit et pour $\varepsilon \leq n h \leq \frac{N+1}{2} h$, on a

$$
\sum_{j=a(h)}^{b(h)} \frac{2 h}{\pi} \sin ^{2}(j n h) \geq \frac{b-a}{2 \pi}
$$

La relation ci-dessus et (24) nous permettent de conclure.

Nous sommes maintenant en mesure d'appliquer le Théorème 2.1. On commence par vérifier la condition $(i)$. Supposons qu'il existe $\left[\begin{array}{l}\varphi_{h} \\ \psi_{h}\end{array}\right] \in X_{h}$ et $\omega \in \mathbb{R}$ tels que

$$
A_{h}\left[\begin{array}{l}
\varphi_{h} \\
\psi_{h}
\end{array}\right]=i \omega\left[\begin{array}{l}
\varphi_{h} \\
\psi_{h}
\end{array}\right]
$$

Alors, en utilisant la définition (15) de $A_{h}$, on obtient aisément que

$$
\left\{\begin{array}{l}
\psi_{h}=i \omega \varphi_{h} \\
{\left[\omega^{2}-A_{0 h}-i \omega\left(B_{0 h} B_{0 h}^{*}\right)\right] \varphi_{h}=0}
\end{array}\right.
$$


En prenant la partie imaginaire du produit scalaire de la seconde relation dans (25) par $\varphi_{h}$ et en utilisant le fait que $A_{0 h}$ est inversible, on obtient que $\varphi_{h}=0$. En utilisant alors la première relation dans (25), il vient que $\psi_{h}=0$. Donc, $i \omega$ ne peut être une valeur propre de $A_{h}$ et donc $i \omega \in \rho\left(A_{h}\right)$ pour tout $\omega \in \mathbb{R}$. Par conséquent, le spectre de l'opérateur $A_{h}$ ne contient pas de points sur l'axe imaginaire et la condition $(i)$ du Théorème 2.1 est donc satisfaite.

Pour démontrer la condition $(i i)$, on raisonne par l'absurde. Supposons donc que pour tout $n \in \mathbb{N}$, il existe $h_{n} \in\left(0, h^{*}\right), \omega_{n} \in \mathbb{R}, z_{n}=\left[\begin{array}{l}\phi_{n} \\ \psi_{n}\end{array}\right] \in X_{h_{n}}$ tel que

$$
\begin{gathered}
\left\|z_{n}\right\|_{X_{h_{n}}}^{2}=\left\|A_{0 h_{n}}^{\frac{1}{2}} \phi_{n}\right\|^{2}+\left\|\psi_{n}\right\|^{2}=1 \quad \forall n \in \mathbb{N} \\
\left\|i \omega_{n} z_{n}-A_{h_{n}} z_{n}\right\|_{X_{h_{n}}} \rightarrow 0 .
\end{gathered}
$$

Pour aboutir à une contradiction, on procède selon les étapes suivantes.

\section{Étape 1}

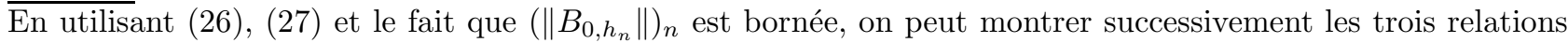
(voir [11] pour les détails)

$$
\begin{gathered}
\left\|B_{0 h_{n}}^{*} \psi_{n}\right\|^{2} \rightarrow 0, \\
\exists \eta>0, \exists n_{0}>0, \forall n>n_{0}, \quad\left|\omega_{n}\right|>\eta, \\
\lim _{n \rightarrow \infty}\left\|A_{0 h_{n}}^{\frac{1}{2}} \phi_{n}\right\|^{2}=\lim _{n \rightarrow \infty}\left\|\psi_{n}\right\|^{2}=\frac{1}{2} .
\end{gathered}
$$

Afin de passer à l'Étape 2, on introduit la décomposition modale de la suite $z_{n}$ sur la base spectrale $\left(\Phi_{m, h_{n}}\right)_{1 \leq|m| \leq N\left(h_{n}\right)}$ de $A_{1 h_{n}}$. Ici, ainsi que dans la suite, on a préféré expliciter pour plus de clarté la dépendance de $N$ vis à vis de $n$ en posant $h_{n}=\frac{\pi}{N\left(h_{n}\right)+1}$. Pour tout $n \in \mathbb{N}$, il existe des coefficients complexes $\left(c_{m}^{n}\right)_{1 \leq|m| \leq N\left(h_{n}\right)}$ tels que

$$
z_{n}=\left[\begin{array}{l}
\phi_{n} \\
\psi_{n}
\end{array}\right]=\sum_{1 \leq|m| \leq N\left(h_{n}\right)} c_{m}^{n} \Phi_{m, h_{n}}
$$

La condition de normalisation (26) s'écrit alors

$$
\sum_{1 \leq|m| \leq N\left(h_{n}\right)}\left|c_{m}^{n}\right|^{2}=1
$$

\section{Étape 2}

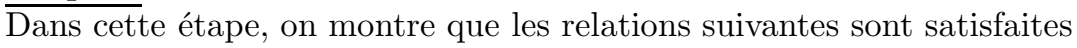

$$
\begin{gathered}
\psi_{n}=\frac{1}{\sqrt{2}} \sum_{m=1}^{N\left(h_{n}\right)}\left(c_{m}^{n}+c_{-m}^{n}\right) \varphi_{m, h_{n}}, \\
\sum_{1 \leq|m| \leq N\left(h_{n}\right)}\left|\omega_{n}-\lambda_{m, h_{n}}\right|^{2}\left|c_{m}^{n}\right|^{2} \rightarrow 0 .
\end{gathered}
$$

La relation (33) s'obtient immédiatement à partir de la seconde composante de (31) en utilisant (19). D'autre part, en utilisant (31) et le fait que $\Phi_{m, h}$ est un vecteur propre de $A_{1 h}$ associé à la valeur propre $i \lambda_{m, h}$, on a

$$
i \omega_{n} z_{n}-A_{1 h_{n}} z_{n}=\sum_{1 \leq|m| \leq N\left(h_{n}\right)} i\left(\omega_{n}-\lambda_{m, h_{n}}\right) c_{m}^{n} \Phi_{m, h_{n}}
$$


La famille $\left(\left\|B_{0 h_{n}}^{*}\right\|\right)$ étant bornée, la relation ci-dessus entraîne (34).

Étape 3

On introduit l'ensemble

$$
\mathcal{F}=\left\{n \in \mathbb{N} \mid \exists m(n) \in \mathbb{Z}^{*}, \text { tel que }\left|\omega_{n}-\lambda_{m(n), h_{n}}\right|<\frac{\gamma}{2}\right\},
$$

où $\gamma$ est défini par (20).

Deux cas sont alors possibles : soit l'ensemble $\mathcal{F}$ est fini et alors il est facile de conclure, ou alors $\mathcal{F}$ est dénombrable et on peut dans ce cas supposer que $\mathcal{F}=\mathbb{N}$. Alors, quitte à réduire si nécessaire la valeur de $\gamma$, on peut supposer que pour tout $m \in \mathbb{Z}^{*}$ avec $m \neq m(n)$, on a

$$
\left|\omega_{n}-\lambda_{m, h_{n}}\right|>\frac{\gamma}{2} .
$$

Posons

$$
\widetilde{\psi}_{n}=\frac{1}{\sqrt{2}} c_{m(n)}^{n} \varphi_{m(n), h_{n}} .
$$

Par (34) on a

$$
\sum_{m \neq m(n)}\left|c_{m}^{n}\right|^{2} \rightarrow 0 .
$$

Les relations (33) de l'Étape 2 et (37) impliquent que

$$
\left\|\psi_{n}-\widetilde{\psi}_{n}\right\| \rightarrow 0 .
$$

Comme $\left(\left\|B_{0 h_{n}}^{*}\right\|\right)$ est bornée, la relation ci-dessus entraîne que

$$
\left\|B_{0 h_{n}}^{*}\left(\psi_{n}-\widetilde{\psi}_{n}\right)\right\| \rightarrow 0
$$

De cette relation et de la relation (28) de l'Étape 1, on déduit que

$$
\left\|B_{0 h_{n}}^{*} \widetilde{\psi}_{n}\right\| \rightarrow 0 .
$$

Utilisant (37), (39) et (21), on obtient finalement que

$$
\psi_{n} \rightarrow 0
$$

ce qui contredit (30).

\section{REFERENCES}

[1] H. T. BANKs, K. Ito, AND C. WANG, Exponentially stable approximations of weakly damped wave equations, in Estimation and control of distributed parameter systems (Vorau, 1990), vol. 100 of Internat. Ser. Numer. Math., Birkhäuser, Basel, 1991, pp. 1-33.

[2] R. Glowinski, C. H. Li, And J.-L. Lions, A numerical approach to the exact boundary controllability of the wave equation. I. Dirichlet controls: description of the numerical methods, Japan J. Appl. Math., 7 (1990), pp. 1-76.

[3] J. A. Infante And E. Zuazua, Boundary observability for the space semi-discretizations of the 1-D wave equation, M2AN Math. Model. Numer. Anal., 33 (1999), pp. 407-438.

[4] L. León AND E. ZuAZuA, Boundary controllability of the finite-difference space semi-discretizations of the beam equation, ESAIM Control Optim. Calc. Var., 8 (2002), pp. 827-862 (electronic). A tribute to J. L. Lions.

[5] K. LIU, Locally distributed control and damping for the conservative systems, SIAM J. Control Optim., 35 (1997), pp. 15741590

[6] K. Liu, Z. Liu, AND B. RAO, Exponential stability of an abstract nondissipative linear system., SIAM J. Control Optimization, 40 (2001), pp. 149-165. 
[7] Z. Liu And S. Zheng, Semigroups associated with dissipative systems, vol. 398 of Chapman \& Hall/CRC Research Notes in Mathematics, Chapman \& Hall/CRC, Boca Raton, FL, 1999.

[8] S. Micu, Uniform boundary controllability of a semi-discrete 1-D wave equation, Numer. Math., 91 (2002), pp. $723-768$.

[9] M. Negreanu and E. Zuazua, Uniform boundary controllability of a discrete 1-D wave equation, Systems Control Lett., 48 (2003), pp. 261-279. Optimization and control of distributed systems.

[10] - Convergence of a multigrid method for the controllability of a 1-d wave equation, C. R. Math. Acad. Sci. Paris, 338 (2004), pp. 413-418.

[11] K. Ramdani, T. Takahashi, And M. Tucsnak, Uniformly exponentially stable approximations for a class of second order evolution equations, ESAIM COCV, à paraître.

[12] K. Ramdani, T. TAKAhashi, AND M. TuCSNAK, Internal stabilization of the plate equation in a square: the continuous and the semi-discretized problems, J. Math. Pures Appl., 85 (2006), pp. 17-37.

[13] L. R. Tcheugoué TéBou and E. Zuazua, Uniform exponential long time decay for the space semi-discretization of a locally damped wave equation via an artificial numerical viscosity, Numer. Math., 95 (2003), pp. 563-598.

[14] E. ZuAzuA, Boundary observability for the finite-difference space semi-discretizations of the 2-D wave equation in the square, J. Math. Pures Appl. (9), 78 (1999), pp. 523-563. 